\title{
Middle East Respiratory Syndrome Coronavirus (MERS-CoV): Perceptions, Predictions, Preventions and the Pilgrimage
}

\section{Rajinder M Joshi*}

Nuclear Medicine and Laboratory Center, Yiaco Medical Co. Al Adan Hospital, Kuwait

The past one year has witnessed the emergence of yet another novel human coronavirus, recently renamed as the Middle East Respiratory Syndrome Corona virus (MERS-CoV) after its first description in 2012 [1,2]. A decade earlier another related virus, the SARS-CoV was responsible for affecting over 8000 people and killing over 800 globally in 2002-2003. The majority of the cases of this new MERS-CoV infection which originated from the Middle East are reported from Kingdom of Saudi Arabia (KSA). However, a few cases have also been reported from Qatar, UAE, Jordan, Tunisia, Italy, Britain, France and Germany mostly in patients who had either travelled to the Kingdom or were referred for further treatment. Interestingly enough only close family contacts, health care providers and other close contacts of the patients have acquired infection to a limited extent. Of the 90 laboratory confirmed cases at the time of writing this article 70 were reported from KSA alone. Out of 45 fatal cases of MERS-CoV infection 38 occurred in KSA. The majority of the cases in the Kingdom originated from $\mathrm{Al}$ Ahsa, its largest Eastern Province.

Corona viruses are widely distributed in animals. Accordingly there are canine, feline, bovine, porcine, avian and murine species prevalent in dogs, cats, cattle, pigs, birds and mice respectively. Corona viruses are single stranded positive sense enveloped RNA viruses, pleomorphic in shape and measure $120-160$ nanometer in diameter and are roughly spherical with a helical nucleocapcid symmetry and have a characteristic fringe of surface projections and hence their name. Some corona viruses may even have an inner fringe of short projections. The virions are sensitive to heat, lipid solvents, non-ionic detergents, oxidizing agents and the UV light. As a rule corona viruses infect only one species, however, a few are capable of infecting closely related animal species. Occasionally they can jump across species. Corona viruses are known for genetic recombinations and hence have the potential for enhanced dissemination and increased infectivity. The human coronaviruses which are known till date include $\mathrm{HCoV}$ 229E, HCoV-OC43, HCoV-HKU 1, HCoV-NL 63, SARS-CoV and the MERS-CoV. They are responsible for majority of the respiratory tract infections but may also cause gastrointestinal tract, renal, hepatic and nervous system infections. While four of the human corona viruses usually cause only common cold like illness, the SARS-CoV and the MERS-CoV are responsible for causing severe and life threatening human infections.

There are speculations that the present MERS-CoV has originated from animals, most likely from bats [3].The molecular analysis has also shown over $98 \%$ homology of MERS-CoV with a bat coronavirus. Whether the recent unprecedented large gatherings of the so called Arab Spring have any significant contribution in the spread of MERS$\mathrm{CoV}$ has neither been proved nor has it been ruled out. However, the aggregation of people usually encourages spread of an infectious disease. After incubation period of a few days to 14 days the MERS$\mathrm{CoV}$ infected patient may have cough, high grade fever, pneumonia and shortness of breath. Rapid kidney failure accounts for fatality in many of the cases. Some patients may have signs and symptoms pertaining to other systems in addition to respiratory tract either because of MERS$\mathrm{CoV}$ or because of co-morbidity. The virus exhibits unique tropism for the nonciliated bronchial epithelial cells (lower respiratory tract) and the kidneys. This may be largely due to carriage of dipeptidyl peptidase 4 receptors (also known as CD 26 receptors) for the virus on these sites $[4,5]$. Furthermore, the virus is equipped with arsenals to elude innate immunity and is capable of antagonizing endogenous interferon. Although, the infections have been seen in people between 2- 94 years of age, the majority of them are encountered in the elderly with preexisting pulmonary, renal, hepatic, metabolic, endocrine disease or an immune compromised state. Over $70 \%$ of the infections are seen in males. The MERS-CoV carries about $50 \%$ of fatality as compared to only about $10-11 \%$ with SARS-CoV infection. The virus can be cultured in the usual cell lines preferably from samples from the lower respiratory tract. RT-PCR may be used for confirming the diagnosis. The immune fluorescence may also be useful but cross reactions with other corona viruses may be encountered. Where ever possible molecular analysis of the strains should be carried out. The general supportive measures form the mainstay of the treatment. The effectiveness of exogenous interferon, protease inhibitors, intravenous immune globulins, ribavirin and corticosteroids has not been conclusively substantiated. Unless properly planned and evaluated in terms of clinical, virological and biomarkers assessment, the above mentioned options are not recommended. However, convalescent plasma (derived from patients recovering from MERS-CoV infection) may be effective in neutralizing the extracellular virions.

The WHO, CDC and other international health organizations are keeping a close and constant watch on the MERS-CoV and are issuing useful updates and guidelines. It is therefore recommended to follow these guidelines to minimize further spread of the virus. Any complacency in this regard may be untenable as much enigma still prevails largely over the epidemiological aspects of the disease. Fortunately, the virus has a low infectivity and requires a close and prolonged contact for the establishment of the infection. But the mere occurrence of about 20 cases of the infection per month should not be the pretext of laxity. Needless to mention that neither the reservoir nor the source/sources of infection are precisely known and our knowledge about the MERS-CoV is still much limited. While the reservoir is likely to be an animal possibly a bat, the sources could be the infected cases or the persons with asymptomatic/subclinical infections or even the environmental objects. The modes and the routes of transmission could also be equally variable. The problems of epidemiological

*Corresponding author: Rajinder M Joshi, Microbiology Unit Radiology, Nuclear Medicine and Laboratory Center, Yiaco Medical Co. Al Adan Hospital, Kuwait, E-mail: drrmjoshi2002@yahoo.com

Received July 28, 2013; Accepted July 31, 2013; Published August 01, 2013

Citation: Joshi RM (2013) Middle East Respiratory Syndrome Coronavirus (MERSCoV): Perceptions, Predictions, Preventions and the Pilgrimage. Clin Microbial 2: e113. doi: 10.4172/2327-5073.1000e113

Copyright: ( 2013 Joshi RM. This is an open-access article distributed under the terms of the Creative Commons Attribution License, which permits unrestricted use, distribution, and reproduction in any medium, provided the original author and source are credited. 
Citation: Joshi RM (2013) Middle East Respiratory Syndrome Coronavirus (MERS-CoV): Perceptions, Predictions, Preventions and the Pilgrimage. Clin Microbial 2: e113. doi: 10.4172/2327-5073.1000e113

investigations on the MERS- $\mathrm{CoV}$ are further compounded by the technical and administrative requirements/formalities in the West, notably the requirement for the screening of animal samples from KSA for Foot and Mouth disease virus. With the Haj pilgrimage period approaching in October when millions of Muslims could visit the Holy Mecca and Medina we need to keep the fingers crossed. Although, the KSA authorities have advised people if possible to postpone their Haj till next year as the expansion work at the facility site is still in process, a significant effect on the reduction of pilgrims may not be achievable. However, if the KSA decides to put restrictions on issuing visas for Haj this year for elderly people and for those with preexisting chronic ailments as a precautionary measure, it will be a significant and a very useful step forward. Though the WHO has not advised any travel ban or restrictions, screening recommendations of the sick people at ports may help minimize spread of infection. In addition to this surveillance the usual preventive measures which may contain further dissemination of MERS-CoV infection include: covering the mouth and nose during coughing and sneezing, disposing the used tissue paper in a trash, no touching of nose and mouth with unwashed hands, washing hands with soap and water or alcohol based antiseptic, avoiding shared utensils, cleaning and disinfection of environmental surfaces, areas, objects, door knobs and handles which are supposed to be contaminated.

There are two main factors which may encourage the spread of MERS-CoV in coming months, one being the congregation of people at Haj and the other the low atmospheric temperature which may favor prolonged viral survival. The herd immunity if already developed against the virus over the past one year may be a significant factor leading to the natural death of the MERS-CoV. Although, nonsustained human to human transmission observation indicates that though sporadic cases or trivial epidemics might occur, the possibility of any major epidemic or a pandemic is virtually ruled out unless the virus has favorable circumstances like large aggregation of people encouraging close prolonged personal contact for dissemination. Any significant mutation in MERS-CoV may equally lead to flare up of the infection.

\section{References}

1. Zaki AM, van Boheemen S, Bestebroer TM, Osterhaus AD, Fouchier RA (2012) Isolation of a novel coronavirus from a man with pneumonia in Saudi Arabia. N Engl J Med 367: 1814-1820.

2. de Groot RJ, Baker SC, Baric RS, Brown CS, Drosten C, et al. (2013) Middle East Respiratory Syndrome Coronavirus (MERS-CoV): Announcement of the Coronavirus Study Group. J Virol 87: 7790-7792.

3. Lu G, Liu D (2012) SARS-like virus in the Middle East: a truly bat-related coronavirus causing human diseases. Protein Cell 3: 803-805.

4. Kindler E, Jónsdóttir HR, Muth D, Hamming OJ, Hartmann R, et al. (2013) Efficient replication of the novel human betacoronavirus EMC on primary human epithelium highlights its zoonotic potential. MBio 4: e00611-00612.

5. Raj VS, Mou H, Smits SL, Dekkers DH, Müller MA, et al. (2013) Dipeptidy peptidase 4 is a functional receptor for the emerging human coronavirus-EMC Nature 495: 251-254. 